\title{
Field emission from multiwall carbon nanotubes on paper substrates
}

\author{
S. M. Lyth ${ }^{\text {a) }}$ and S. R. P. Silva \\ Nanoelectronics Centre, Advanced Technology Institute, University of Surrey, Guildford, \\ Surrey GU2 7XH, United Kingdom
}

(Received 30 November 2006; accepted 2 April 2007; published online 27 April 2007)

\begin{abstract}
The authors report extremely low electron field emission thresholds from acid oxidized multiwall carbon nanotubes deposited on paper substrates by dip coating in an aqueous nanotube ink. Using paper substrates of differing surface roughness, field emission threshold fields ranging from 0.8 to $11.6 \mathrm{~V} / \mu \mathrm{m}$ were observed, varying in an approximate inverse linear log relationship with the surface roughness of the underlying paper substrate. This study shows how field emission from supported nanotube films can be tailored via the morphology of the scaffold substrate, and how these composite electrodes can be straightforwardly fabricated on cheap, flexible substrates. (C) 2007 American Institute of Physics. [DOI: 10.1063/1.2734379]
\end{abstract}

Carbon nanotubes (CNTs) are known for their excellent field emission properties ${ }^{1-4}$ owing to their large aspect ratio and high electrical conductivity. ${ }^{5}$ They also possess high mechanical integrity and chemical inertness. Consequently, CNTs have been extensively investigated as cold cathodes for lighting and flat panel displays. ${ }^{6-9}$ The realization of low cost, large area CNT cathodes is crucial if these devices are to become contenders in the marketplace. Several groups have experimented with field emitting inks and inkjet printing of CNTs for electronics applications. ${ }^{10-15}$ Ink jet printing, dip coating, screen printing, and processing on flexible substrates all lend themselves to industrialization and continuous manufacturing processes, with obvious cost benefits over vacuum deposition, thermal evaporation, or chemical vapor deposition.

The morphology of a field emitting surface plays a crucial role in determining its field emission properties. ${ }^{16,17}$ It is the local field at the emission site that governs emission, so micron and nanoscale protrusions can greatly enhance the emission due to increased field concentration above their surfaces. This enhancement is generally described by the field enhancement factor $(\beta)$, which is the factor by which the local electric field exceeds the macroscopic applied electric field. Therefore, by altering the morphology of an emitter, $\beta$ and consequently the field emission properties would be expected to vary.

In this letter, we present a method for homogeneously coating paper substrates with multiwall carbon nanotubes (MWNTs) via a straightforward dip coating technique. The field emitting properties of these substrates were investigated as a function of the surface roughness (and thereby the morphology) of the underlying paper substrate. This technique brings about the possibility of directly depositing nanotubes in a controlled manner onto a plethora of prepatterned substrates with surface morphology chosen to maximize $\beta$.

The graphitic surface of MWNTs renders them hydrophobic and it is notoriously difficult to obtain a stable dispersion in common organic solvents without chemical derivatization of the outer surface. Stable aqueous dispersions are commonly formed by refluxing MWNTs in a concentrated nitric and sulfuric acid mixture, resulting in the forma-

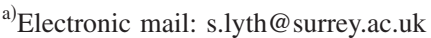

tion of oxygen containing moieties including carboxylic acid groups attached to the MWNT ends and sidewalls which interact with water molecules via hydrogen bonding. ${ }^{15}$ Acid treatment also serves to purify MWNTs removing catalytic particles and amorphous carbon. For the sake of clarity, acid oxidized MWNTs will herein be referred to as $o$-MWNTs.

High purity $(95 \%)$ MWNTs with a nominal diameter of $10 \mathrm{~nm}$ were purchased from Nanocyl and ultrasonically dispersed in a 3:1 concentrated sulfuric and nitric acid mixture for 10 mins. The mixture was refluxed at $110^{\circ} \mathrm{C}$ for $40 \mathrm{~min}$ before diluting with de-ionized water. Large particles and agglomerates were removed from the solution by centrifuging. The centrifuged liquid was carefully decanted, filtered through a $0.2 \mu \mathrm{m}$ polycarbonate membrane, and washed with de-ionized water until a slurry with $p \mathrm{H} \mathrm{6-7}$ was obtained. This slurry was added to a small amount of deionized water, which was sonicated to disperse the tubes. The resulting product was a stable dispersion of $o$-MWNTs. The $o$-MWNT loading $(3.5 \mathrm{mg} / \mathrm{ml})$ was determined by drying and weighing a portion of the $o$-MWNT ink. A variety of different grades of commercially available paper was dipped into the $o$-MWNT ink, removed and baked at $150{ }^{\circ} \mathrm{C}$ for $10 \mathrm{~min}$ to remove residual water. This resulted in a thick, black coating of $o$-MWNTs on the paper's surface.

A profilometer was used to measure the average surface roughness $\left(R_{A}\right)$ of the surfaces of different types of paper, which varied from 1.3 to $9.8 \mu \mathrm{m}$. Measurements were carried out over a $2 \mathrm{~mm}$ scan length, and an average of ten measurements across the substrate surface $\left(\sim 1 \mathrm{~cm}^{2}\right)$ were performed.

Scanning electron microscopy of the various substrates is presented in Fig. 1. Figures 1(a)-1(c) show the morphology of the paper scaffold with the lowest surface roughness after the $o$-MWNT dip coating process. Figures 1(d)-1(f) show the morphology of the paper substrate found to have the largest surface roughness. The difference between the two samples is clear; the sample with low $R_{A}$ has relatively few, widely spaced surface features, and the substrate with high $R_{A}$ comprises large, closely packed protruding features. Both samples show $100 \%$ surface coverage by the $o$-MWNT ink.

The field emission properties of the paper substrates were investigated at $10^{-6}$ mbar in a simple diode configuration, with a 5-mm-diameter spherical stainless steel anode. 


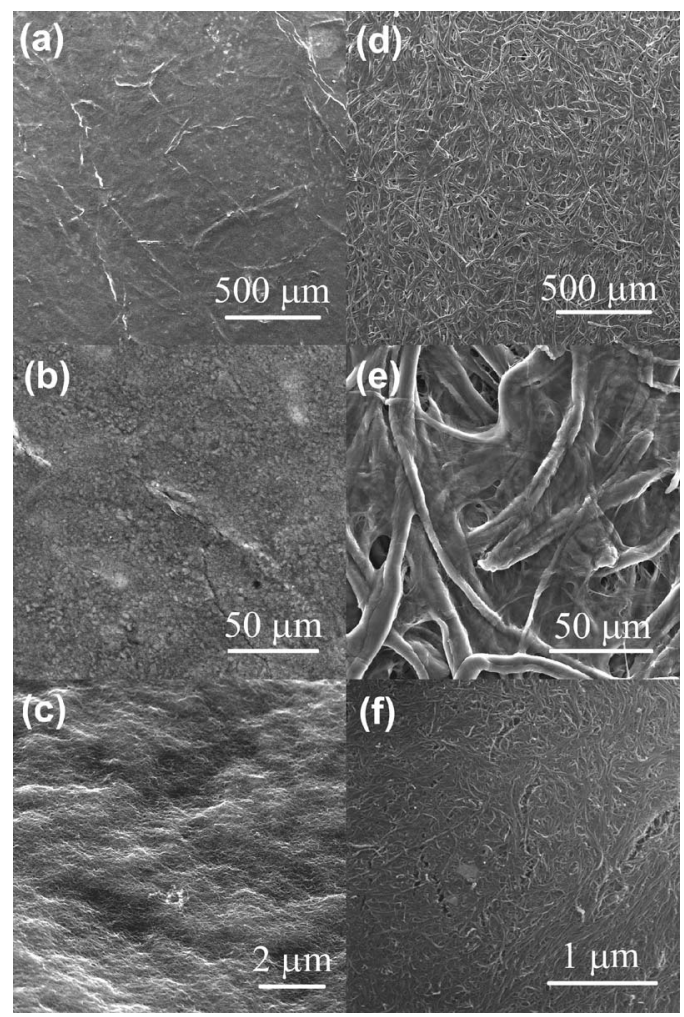

FIG. 1. SEM images of $[(\mathrm{a})-(\mathrm{c})]$ low roughness paper substrate coated with $o$-MWNT ink. [(d)-(f)] High roughness paper substrate coated with $o$-MWNT ink.

Electrical connections were made via top contacts of silver paste at the edges of the paper substrate. The anode voltage (dc) was incremented in $30 \mathrm{~V}$ steps. The macroscopic electric field (E) was calculated by dividing the applied voltage by the electrode gap, typically $200 \mu \mathrm{m}$. The threshold field $\left(E_{\mathrm{th}}\right)$ was defined as the macroscopic electric field at which an emission current of $1 \mathrm{nA}$ was detected. The average $E_{\text {th }}$ was calculated over six widely spaced emission sites on each sample, with the voltage cycled up and down five times at each site.

Field emission data are presented in Table I and plotted in Fig. 2. Figure 2(a) shows emission current versus macroscopic electric field for all the substrates. The threshold field was observed to vary from 0.8 to $11.6 \mathrm{~V} / \mu \mathrm{m}$. It should be noted that very little hysteresis is observed between the up and down cycles and that no initial conditioning was observed in the first cycle. Fowler Nordheim graphs ${ }^{18}$ [Fig. 2(b)] were plotted using the current-field data and the enhancement factors $(\beta)$ of the different samples were calculated from the slopes, taking the work function to be $5.0 \mathrm{eV} .{ }^{19} \mathrm{~A}$ large difference between the slopes of the plots

TABLE I. Summary of numerical results.

\begin{tabular}{cccc}
\hline \hline $\begin{array}{c}\text { Surface roughness } \\
(\mu \mathrm{m})\end{array}$ & $\begin{array}{c}\text { Threshold field } \\
(\mathrm{V} / \mu \mathrm{m})\end{array}$ & $\begin{array}{c}\text { Hysteresis at } \\
1 \mathrm{nA}(\mathrm{V} / \mu \mathrm{m})\end{array}$ & $\begin{array}{c}\text { Enhancement factor } \\
(\beta)\end{array}$ \\
\hline $1.3 \pm 0.3$ & $11.6 \pm 3.9$ & 1.9 & $474 \pm 17$ \\
$2.4 \pm 0.3$ & $6.0 \pm 0.3$ & 0.6 & $667 \pm 17$ \\
$3.4 \pm 0.7$ & $7.5 \pm 1.8$ & 0.7 & $564 \pm 20$ \\
$4.4 \pm 1.1$ & $4.9 \pm 1.1$ & 0.4 & $934 \pm 13$ \\
$4.9 \pm 0.8$ & $1.5 \pm 0.2$ & 0.2 & $3910 \pm 97$ \\
$9.8 \pm 1.0$ & $0.8 \pm 0.2$ & 0.05 & $4740 \pm 86$ \\
\hline \hline
\end{tabular}
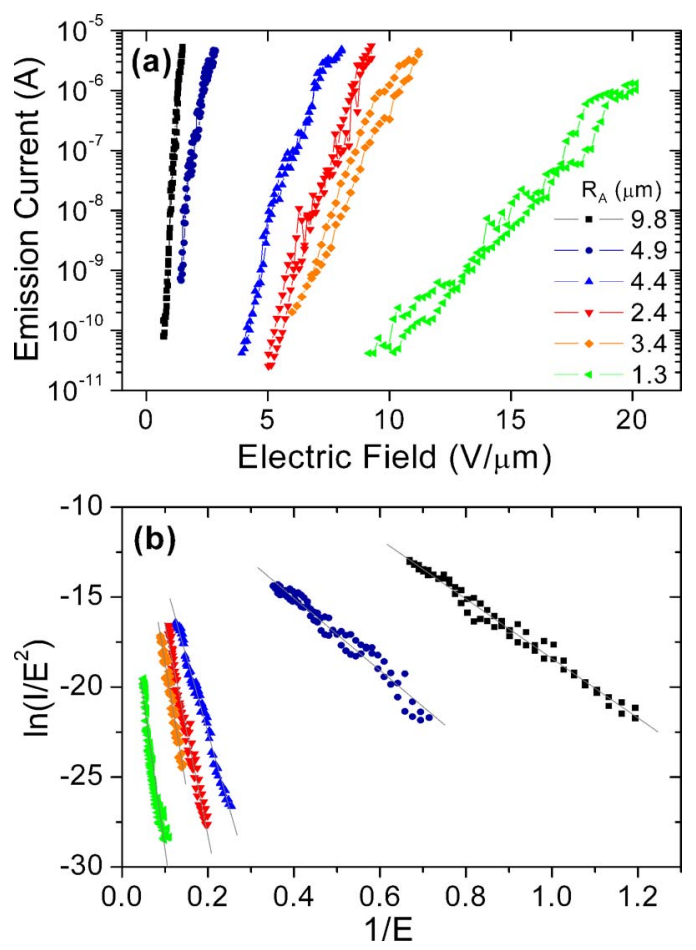

FIG. 2. (Color online) (a) Comparison of field emission current-field curves for $o$-MWNT coated paper of varying surface roughness. (b) FowlerNordheim plots of field emission data.

can easily be observed, resulting in a wide range in $\beta$ from $\sim 500$ for relatively smooth paper $\left(R_{A}=1.3 \mu \mathrm{m}\right)$ to $\sim 5000$ for the roughest paper $\left(R_{A}=9.8 \mu \mathrm{m}\right)$.

These extremely low threshold fields and high enhancement factors are attributed to the fact that the underlying paper substrates have protruding features with high aspect ratios, contributing to a high geometric field enhancement and therefore high local fields at the emission sites. The very high values of $\beta$ could be partly due to a giant multistage geometric enhancement effect arising from the product of the separate enhancement factors of the paper substrate and the $o$-MWNT coating. ${ }^{20}$

Figure 3 shows $E_{\text {th }}$ as a function of $R_{A}$ for the samples. A glass slide spin coated with $o$-MWNT was also characterized to represent a "flat" zero point on the roughness scale $\left(R_{A}\right.$

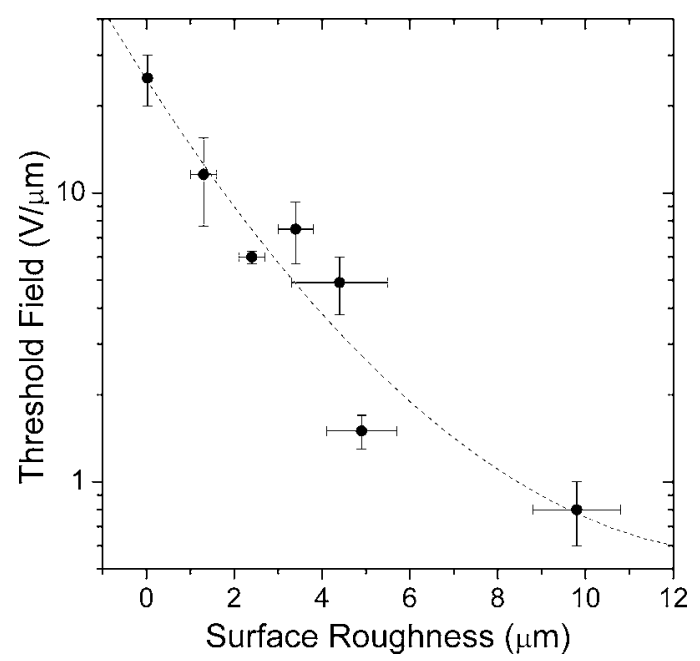

FIG. 3. Plot of threshold field $\left(E_{\mathrm{th}}\right)$ vs surface roughness $\left(R_{A}\right)$ for $o$-MWNT coated paper substrates. 
$=23 \mathrm{~nm}$ ). A clear trend can be observed, where the field emission characteristics improve as $R_{A}$ increases. This can be related to the morphology of the films: the smoothest films have relatively few surface features [Fig. 1(a)], therefore having a very low geometric field enhancement factor, resulting in relatively high threshold fields. The roughest films have an uneven morphology with many surface features [Fig. 1(c)] and therefore a high geometric field enhancement factor, resulting in low threshold fields. The statistical error in the measurements can be seen on the error bars in Fig. 3, though this clearly does not account for all the errors. This is probably due to the fact that using $R_{A}$ as a gauge of surface morphology is a simplistic approach. Other factors such as the aspect ratios of surface features, curvature at surfaces, and the specific morphology of the substrates are clearly important, but difficult to combine and describe numerically on samples with such complex surfaces.

In conclusion, flexible field emitters were fabricated with a range of extremely low turn on fields $(<1 \mathrm{~V} / \mu \mathrm{m})$ and high geometric field enhancement factors on insulating, flexible substrates. A trend of improved field emission characteristics with increased surface roughness was observed. The wide range in $E_{\text {th }}$ and $\beta$ show how relatively straightforward it is to tailor field emission characteristics using simple fabrication techniques. This technique provides a cheap, low temperature method of manufacturing efficient field emitters via inkjet printing, dip coating, or screen printing onto readily available, flexible substrates.

The authors would like to thank EPSRC (UK) for funding this research via the CBE and Portfolio Partnership programs. Thanks are due to Ross Hatton for helpful discussion.

${ }^{1}$ W. A. Deheer, A. Chatelain, and D. Ugarte, Science 270, 1179 (1995).
${ }^{2}$ A. G. Rinzler, J. H. Hafner, P. Nikolaev, L. Lou, S. G. Kim, D. Tomanek, P. Nordlander, D. T. Colbert, and R. E. Smalley, Science 269, 1550 (1995).

${ }^{3}$ W. B. Choi, D. S. Chung, J. H. Kang, H. Y. Kim, Y. W. Jin, I. T. Han, Y. H. Lee, J. E. Jung, N. S. Lee, G. S. Park, and J. M. Kim, Appl. Phys. Lett. 75, 3129 (1999).

${ }^{4}$ R. C. Smith, J. D. Carey, C. H. P. Poa, D. C. Cox, and S. R. P. Silva, J. Appl. Phys. 95, 3153 (2004).

${ }^{5}$ R. H. Baughman, A. A. Zakhidov, and W. A. Deheer, Science 297, 787 (2002).

${ }^{6}$ J. M. Bonard, T. Stockli, F. Maier, W. A. de Heer, A. Chatelain, J. P. Salvetat, and L. Forro, Phys. Rev. Lett. 81, 1441 (1998).

${ }^{7}$ Q. H. Wang, A. A. Setlur, J. M. Lauerhaas, J. Y. Dai, E. W. Seelig, and R. P. H. Chang, Appl. Phys. Lett. 72, 2912 (1998).

${ }^{8}$ R. C. Smith, D. C. Cox, and S. R. P. Silva, Appl. Phys. Lett. 87, 103112 (2005).

${ }^{9}$ S. M. Lyth, R. A. Hatton, and S. R. P. Silva, Appl. Phys. Lett. 90, 013120 (2007).

${ }^{10}$ K. Krisztian, T. Mustonen, G. Toth, H. Jantunen, M. Lajunen, C. Soldano, S. Talapatra, S. Kar, R. Vajita, and P. M. Ajayan, Small 2, 1021 (2006).

${ }^{11}$ J. Wang and M. Musameh, Analyst (Cambridge, U.K.) 129, 1 (2004).

${ }^{12}$ Z. J. Fan, T. Wei, G. H. Luo, and F. Wei, J. Mater. Sci. 40, 5075 (2005).

${ }^{13}$ H. Y. Shin, W. S. Chung, K. H. Kim, Y. R. Cho, and B. C. Shin, J. Vac. Sci. Technol. B 23, 2369 (2005).

${ }^{14}$ A. P. Burden, H. E. Bishop, M. Brierley, J. M. Friday, C. Hood, P. G. A. Jones, A. Y. Khazov, W. Lee, R. J. Riggs, V. L. Shaw, and R. A. Tuck, J. Vac. Sci. Technol. B 18, 900 (2000).

${ }^{15}$ H. Ago, T. Kugler, F. Cacialli, W. R. Salaneck, M. S. P. Shaffer, A. H. Windle, and R. H. Friend, J. Phys. Chem. B 103, 8116 (1999).

${ }^{16}$ L. Nilsson, O. Groening, C. Emmenegger, O. Kuettel, E. Schaller, L. Schlapbach, H. Kind, J. M. Bonard, and K. Kern, Appl. Phys. Lett. 76, 2071 (2000).

${ }^{17}$ R. C. Smith, R. D. Forrest, J. D. Carey, W. K. Hsu, and S. R. P. Silva, Appl. Phys. Lett. 87, 013111 (2005).

${ }^{18}$ R. H. Fowler and L. Nordheim, Proc. R. Soc. London, Ser. A 119, 173 (1928).

${ }^{19}$ N. P. Blanchard, R. A. Hatton, and S. R. P. Silva, Chem. Phys. Lett. 434, 92 (2006).

${ }^{20}$ J. Y. Huang, K. Kempa, S. H. Jo, S. Chen, and Z. F. Ren, Appl. Phys. Lett. 87, 053110 (2005). 